\title{
Expression of toll-like receptor 4 gene polymorphism and inflammatory factors in peripheral blood on patients with sepsis
}

\author{
Tieying Tian, Xiyuan Xu, Jingping Yang* \\ Department of Respiratory and Critical Medicine, the Third Affiliated Hospital of Inner Mongolia Medical University (Baogang \\ Hospital), Baotou, Inner Mongolia, China
}

Received: December 12, 2020

Accepted: April 10, 2021

Online Published: April 26, 2021

DOI: $10.5430 /$ dcc.v7n4p15

URL: https://doi.org/10.5430/dcc.v7n4p15

\begin{abstract}
Objective: To observe the presence of toll-like receptor 4 (TLR4) gene polymorphism in the venous blood in patients with sepsis, meanwhile, to observe the expression and the diagnostic value of TLR4 mRNA, interferon- $\gamma$ (IFN- $\gamma$ ), interleukin-23 (IL-23), procalcitonin (PCT) and C-reactive protein (CRP) in patients with different degrees of sepsis.

Methods: The peripheral blood samples from the subjects were collected to extract genomic DNA, gene sequencing and enzyme digestion method were applied to the detection of TLR4 Asp299Gly loci polymorphism. The expression of TLR4 mRNA in the peripheral blood was detected by reverse transcriptase polymerase chain reaction (RT-PCR). The expression levels of IFN- $\gamma$, IL-23, PCT and CRP were measured by enzyme linked immunosorbent assay (ELISA).

Results: (1) The gene polymorphism was not present in TLR4 Asp299Gly loci; (2) The expression of TLR4 mRNA in the peripheral blood in patients with sepsis: on $\mathrm{d} 1$, there were statistically significant differences between the normal group and the group (APACHE II $\leq 20$ ), between the normal group and the group (APACHE II $>20$ ), between the group (APACHE II $\leq 20$ ) and the group (AAPACHE II $>20)(t=5.741,14.780$ and 10.500, all $p<.01)$. APACHE II stands for Acute Physiology and Chronic Health Evaluation II. On d7, there were statistically significant differences between the normal group and the group (APACHE II $\leq 20$ ), between the normal group and the group (APACHE II $>20$ ), between the group (APACHE II $\leq 20$ ) and the group (APACHE II $>20)(t=4.186,13.830$ and 9.645, all $p<.01)$; (3) The expression levels of IFN- $\gamma$, IL-23, PCT and CRP were obviously upregulated (all $p<.01$ ), and TLR4 was positively correlated with IFN- $\gamma$ and IL-23 (all $p<.01$ ); (4) The best cutoff value of TLR4 mRNA at baseline was $891.6 \mu \mathrm{g} / \mathrm{L}$, with a sensitivity of $100 \%$ and a diagnostic specificity of $57 \%$. The best cutoff value of IFN- $\gamma$ at baseline was $84.5 \mu \mathrm{g} / \mathrm{L}$, with a sensitivity of $100 \%$ and a diagnostic specificity of $57 \%$. The best cutoff value of IL-23 at baseline was $861 \mu \mathrm{g} / \mathrm{L}$, with a sensitivity of $100 \%$ and a diagnostic specificity of $97 \%$.

Conclusions: (1) The Asp299Gly polymorphism is not present in TLR4 gene; (2) The expression levels of TLR4 mRNA, IFN- $\gamma$ and IL-23 are relatively high in patients with sepsis, and will be higher with the increased severity of sepsis; (3) TLR4 mRNA, IFN- $\gamma$ and IL-23 can be used as molecular biomarkers for the early diagnosis of sepsis.
\end{abstract}

Key Words: Sepsis, Toll-like receptor 4, Interleukin-23, Gene polymorphism

\footnotetext{
*Correspondence: Jingping Yang; Email: yangron@sina.com.cn; Address: Department of Respiratory and Critical Medicine, the Third Affiliated Hospital of Inner Mongolia Medical University (Baogang Hospital), Baotou, Inner Mongolia 014010, China.
} 


\section{INTRODUCTION}

Sepsis is an organ dysfunction resulting from response disorder to infection and a threat to life. With the further development of disease, it can lead to septic shock and multiple organ dysfunction syndrome. It is one of the most commonly seen problems to be solved by the high level in the area of critical diseases. ${ }^{[1]}$ Even though the levels of understanding and clinical settlement of sepsis have been obviously improved, the incidence and the morality of sepsis are not reduced accordingly, which becomes a crucial medical problem at the present stage. ${ }^{[2]}$ In the early stage of sepsis, it is difficult to determine the severity of disease by clinical manifestations and objective indicators, which directly affects the outcome. ${ }^{[3]}$ Therefore, it is of great value to the prognosis to look for new biomarkers used in the early diagnosis of sepsis and the determination of the severity. Some researches have shown that innate and adaptive immune responses have a particularly important relationship with gene regulation. ${ }^{[3-8]}$ Toll-like receptors (TLRs) play an important role in innate immune responses. It can activate inflammatory signal transduction pathway by identifying pathogenic molecules and binding to the corresponding receptors. ${ }^{[3,4]}$ TLR4, a member of TLR family, is an important lipopolysaccharide (LPS) ligand which participate in the generation of inflammatory factors. ${ }^{[3,4]}$ Single nucleotide polymorphisms (SNPs) are present in TLR4. Different SNPs have an influence on the regulation and progression of TLR4 to the immune inflammatory responses. ${ }^{[3,4]}$ Currently, the researches on TLR4-related SNPs are mainly focused on Asp299Gly loci of non-Asian population. ${ }^{[3,4]}$ Hence, this research is designated to understand the SNPs present in Asp299Gly loci on TLR4 genes in sepsis patients of the Han nationality and define the significance in the diagnosis of sepsis by measuring different expression levels of TLR4 mRNA and inflammatory factors in the patients with different degree of sepsis.

\section{OBJECTS AND METHODS}

\subsection{Research objects}

The patients (of Han nationality) admitted in Department of Respiratory and Critical Medicine of Baogang Hospital of Inner Mongolia from August 2014 to November 2017 were selected and diagnosed according to SEPSIS 3.0 diagnostic standards. ${ }^{[1]}$ Exclusion criteria: (1) patients with acquired immunodeficiency syndrome; (2) patients who were given the treatment with immunosuppressants after organ transplantation; (3) patients who used glucocorticoids within the previous 4 weeks; (4) patients who received chemotherapy within the previous 8 weeks; (5) patients who were less than 18 years old; (6) patients who withdrew from the research due to against-advice discharge. Grouping: according to Acute Physiology and Chronic Health Evaluation II (APACHE II), these patients were divided into three groups: the group (APACHE II $\leq 20$ ), in which 94 cases of patients ( 54 cases of male and 40 cases of female) with APACHE II $\leq 20$, aged from 55 to 78, with an average age of $64.9 \pm 7.4$; the group (APACHE II $>20$ ), in which 68 cases of patients (38 cases of male and 30 cases of female) with APACHE II $>20$, aged from 50 to 75 , with an average age of $63.8 \pm 7.2$; the normal group, in which 62 cases of the healthy adults ( 37 cases of male and 25 cases of female) receiving the physical examination in Heath Medical Center of our hospital were randomly selected without recent infection history during the same period, aged from 50 to 75 , with an average age of $64.5 \pm 7.5$. There were no statistically significant differences in gender and age among all the subjects $(p>.05)$. This research was in conformity with the principles established by Declaration of Helsinki.

\subsection{Research methods}

\subsubsection{Sample collecting}

It was required to collect $4 \mathrm{ml}$ of venous blood sample from each subject respectively on $\mathrm{d} 1$ and $\mathrm{d} 7$ after admission to the hospital. Each blood sample was divided into two tubes. The first tube of $2 \mathrm{ml}$ blood sample was centrifuged at the rotate speed of 3,000 r/min for $5 \mathrm{~min}$ (centrifugal radius: 10 $\mathrm{cm}$ ) after autoagglutination. The second tube of $2 \mathrm{ml}$ blood sample was added by $2 \%$ EDTA for anti-coagulation. All samples were treated with gradient freezing and placed in a $-80^{\circ} \mathrm{C}$ refrigerator for the use of batch measurement.

\subsubsection{The measurement of SNPs in TLR4 gene}

Blood Gen Mini Kits (CoWin Biosciences, Product No.: CW2087) were applied to the extraction of DNA, and the automatic microplate reader (Thermo Fisher Scientific, USA) was used to measure the concentration of sample DNA. It was required to amplify Asp299Gly loci in TLR4 gene (PCR, manufactured by ABI in USA). DNA sequence was required to be looked up via http://www.ncbi.nlm.nih .gov/projects/SNP/ for primer designing. Primer 5.0 primer designing software was used to design primers for the targeted genes. The primers in this research were made up of forward primers and reverse primers. Primer sequence was: TLR4 Asp299Gly; the upstream primer was: 5'-ATACTTAGACTACTACCTCCATG-3'; the downstream primer: 5'-TTGTTGGAAGTGAAAGTAAG-3'. TIANgel Midi Purification Kits (Tiangen Biotech (Beijing) Co., Ltd., Product No.: DP209) were used to purify TLR4 Asp299Gly, restriction endonuclease Nco I was applied to enzyme digestion, and the site was Nco I: 5'... C $\nabla$ TCATGG...3'; 3'...GGTAC $\nabla$ C...5'. Agarose gel electrophoresis (Bio- 
Rad) was used to acquire enzyme-digested products and then analyze restriction fragment length polymorphism (taking DNA Marker I as relative molecular weight standard).

\subsubsection{Gene sequencing}

Gene sequencing was conducted towards the purified PCR products (BGI).

\subsubsection{The measurement of TLR4 mRNA in the peripheral blood}

According to the instructions, the reverse-transcription of PCR was made to extract the total RNA of a single nucleus and then Real Time PCR was performed to measure the expression of TLR4 mRNA (PCR amplifier, manufactured by ABI of USA). It was required to look up complete sequenced protein coding genes of TLR4 on NCBI gene bank for primer designing. The primers contained: NR-H-TLR4-upstream primer, NR-H-TLR4-downstream primer, TH-GAPDH-upstream primer and TH-GAPDHdownstream primer. In addition, the primer sequences were as follows: 5'-CATTGGTGTGTCGGTCCTCA-3', 5' CCAGTCCTCATCCTGGCTTG-3', 5'-CCAGGTGGTCTC CTCTGACTTC-3' and 5'-GTGGTCGTTGAGGGCAATG3'. $\Delta \mathrm{Ct}$ method was used to calculate the quantitative results (Mean relative content $\%=$ $2^{-m e a n \Delta C t} ; \Delta \mathrm{Ct}_{\text {unknown sample }}=\mathrm{Ct}_{\text {unknown sample }}-$ $\left.\mathrm{Ct}_{\text {internal reference }}\right)$. The internal reference was considered as GAPDH.

\subsubsection{The measurement of the levels of IFN- $\gamma, I I-23, P C T$ and CRP in the serum}

Enzyme linked immunosorbent assay (ELISA) was made strictly following the instruction.

\subsection{Statistical analysis}

SPSS 13.0 statistical software was applied to statistical calculation, and the experimental data were represented by $\bar{X} \pm$ S. ANOVA was applied to the comparison among different groups of data. If the variance was homogeneous, one way ANOVA was applied; if not, rank sum test was used in the comparison. The correlation was analyzed by use of linear correlation analysis, and receiver operating characteristic (ROC) curve was applied to the analysis in the diagnostic sensitivity and specificity of sepsis. The difference $p<.05$ was of statistical significance. If SNPs were present, then Hardy-Weinberg equilibrium (HWE) was conducted to genetic frequency. HaPloview 4.2 software was used to conduct the chi-square test towards genotype distribution and allele frequency [(two-sided test, $\alpha=0.05$ ), and calculate the odds ratio (OR) of allele frequency and $95 \%$ confidence interval].

\section{Results}

\subsection{Enzyme digestion results of TLR4 Asp299Gly}

After TLR4 Asp299Gly loci were treated with Nco I enzyme digestion, it was found that the PCR products of TLR4 Asp299Gly were $218 \mathrm{bp}$, and the gene locus mutation was not seen (see Figure 1).

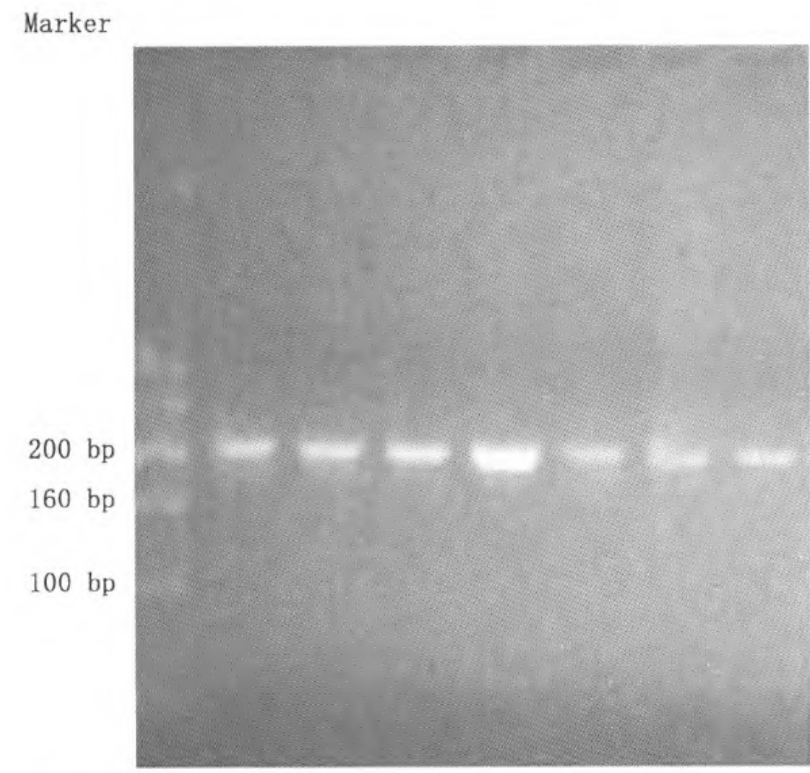

Figure 1. Electrophoretogram of Nco I enzyme digestion products of TLR4 Asp299Gly DNA Marker 20 is used; TLR4 stands for toll-like receptor 4

\subsection{The results of gene sequencing}

The gene sequencing was conducted to the purified products before enzyme digestion, it was found that the peak map of gene sequencing was well shown and the gene locus mutation (TLR4 Asp299Gly) was not seen (see Figure 2).

\subsection{The expression of TLR4 mRNA in the peripheral blood (detected by RT-PCR)}

The results showed that on $\mathrm{d} 1$, the expression levels of TLR4 mRNA in the normal group, the group (APACHE II $\leq 20$ ) and the group (APACHE II $>20$ ) were $816 \pm 162,1,324 \pm$ 231 and 2,124 \pm 639 . Compared with the normal group, the expression levels of TLR4 mRNA in the group (APACHE II $\leq 20$ ) and the group (APACHE II $>20$ ) were obviously higher $(t=9.000,22.000$; all $p<.0001)$; the expression level of TLR4 mRNA in the group (APACHE II > 20) was obviously higher than that in the group (APACHE II $\leq 20)(t$ $=15.000, p<.0001)$. On d7, the expression levels of TLR4 mRNA in the normal group, the group (APACHE II $\leq 20$ ) and the group (APACHE II > 20) were $822 \pm 167,886 \pm$ 206 and 1,097 \pm 448 . The expression level of TLR4 mRNA in the group (APACHE II > 20) was obviously higher than 
that in the normal group and the group (APACHE II $\leq 20$ ) $(t=4.500,3.830$; all $p<.01)$. There was no statistically significant difference between the normal group and the group (APACHE II $\leq 20)(t=1.100, p>.05)$. The expression levels of TLR4 mRNA in the group (APACHE II $\leq 20$ ) and the group (APACHE II $>20$ ) on $\mathrm{d} 7$ were both lower than those on $\mathrm{d} 1(t=8.700,17.000$; all $p<.0001)$. See Figure 3 for details.

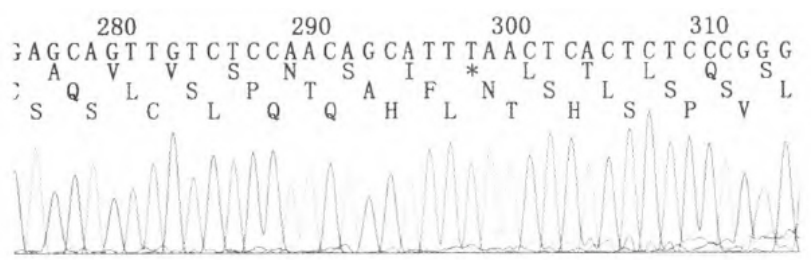

Figure 2. TLR4 (Asp299Gly) gene sequencing peak map TLR4 stands for toll-like receptor 4

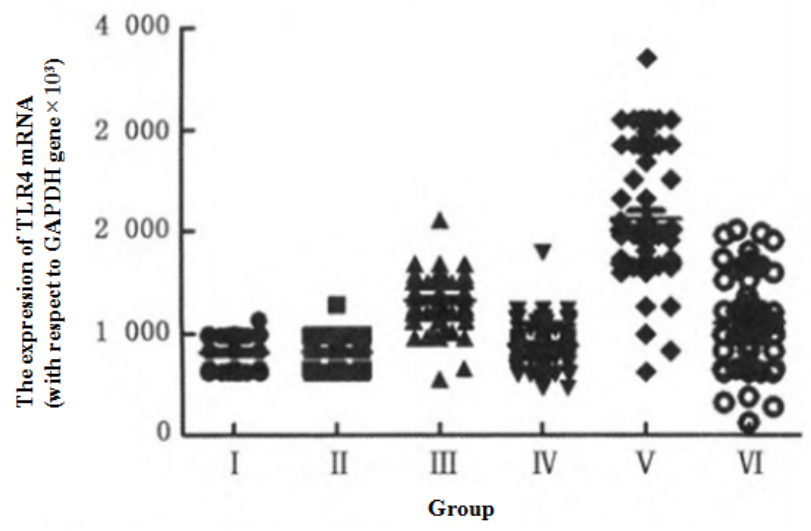

Figure 3. The expression of TLR 4 mRNA in each group of PBMC

I stands for the normal group on d1; II stands for the normal group on d7; III stands for the group (APACHE II $\leq 20)$ on 11 ; IV stands for the group (APACHE II $\leq 20)$ on $d 7 ; V$ stands for the group (APACHE II > 20) on d1; VI stands for the group (APACHE II > 20) on d7; PBMC is for peripheral blood mononuclear cell; TLR4 is for toll-like receptor 4 .

\subsection{The expression of IFN- $\gamma$, IL-23 and PCT in the} serum by means of ELSIA method

On $\mathrm{d} 1$, the expression levels of IFN- $\gamma$ in the normal group, the group (APACHE II $\leq 20$ ) and the group (APACHE II $>20)$ were $(107.9 \pm 47.01) \mu \mathrm{g} / \mathrm{L},(183.3 \pm 62.53) \mu \mathrm{g} / \mathrm{L}$ and $(252.8 \pm 83.52) \mu \mathrm{g} / \mathrm{L}$ respectively; the expression levels of IL-23 were $(501 \pm 223) \mu \mathrm{g} / \mathrm{L},(1,957 \pm 657) \mu \mathrm{g} / \mathrm{L}$ and $(2,980 \pm 713) \mu \mathrm{g} / \mathrm{L}$ respectively; the expression levels of PCT were $(0.25 \pm 0.06) \mu \mathrm{g} / \mathrm{L},(2.62 \pm 4.03) \mu \mathrm{g} / \mathrm{L}$ and $(5.97$ $\pm 6.66) \mu \mathrm{g} / \mathrm{L}$. On $\mathrm{d} 1$, the above-mentioned indicators in the group (APACHE $\mathrm{II} \leq 20$ ) and the group (APACHE II $>20$ ) were obviously higher than those in the normal group $(t=$
7.564 and 13.550, 19.000 and 31.800, 4.020 and 9.010; all $p$ $<.01$ ); the indicators in the group (APACHE II > 20) were obviously higher than those in the group (APACHE II $\leq 20$ ) $(t=13.550,14.500,5.810$; all $p<.01)$.

On $\mathrm{d} 7$, the expression levels of IFN- $\gamma$ in the normal group, the group (APACHE II $\leq 20$ ) and the group (APACHE II > 20) were $(105.5 \pm 47.01) \mu \mathrm{g} / \mathrm{L},(108.3 \pm 47.46) \mu \mathrm{g} / \mathrm{L}$ and $(154.9 \pm 71.05) \mu \mathrm{g} / \mathrm{L}$ respectively; the expression levels of IL-23 were $(499 \pm 217) \mu \mathrm{g} / \mathrm{L},(536 \pm 252) \mu \mathrm{g} / \mathrm{L}$ and $(890$ $\pm 359) \mu \mathrm{g} / \mathrm{L}$ respectively; and the expression levels of PCT were $(0.25 \pm 0.06) \mu \mathrm{g} / \mathrm{L},(0.99 \pm 1.11) \mu \mathrm{g} / \mathrm{L}$ and $(3.18 \pm$ 4.17) $\mu \mathrm{g} / \mathrm{L}$ respectively.

On $\mathrm{d} 7$, there were no statistically significant differences in the above-mentioned indicators between the normal group and the group (APACHE $\mathrm{II} \leq 20)(t=0.2884,0.490$ and 1.249; all $p>.05)$. However, these indicators in the group (APACHE II $>20$ ) were still higher than those in the normal group $(t=4.619,4.800$ and 4.606; all $p<.01)$. These indicators in the group (APACHE II $>20$ ) were obviously higher than those in the group (APACHE II $\leq 20)(t=4.619,4.800$ and 4.606; all $p<.01)$.

The expression levels of IFN- $\gamma$, IL-23 and PCT in the group (APACHE II $\leq 20$ ) and the group (APACHE II > 20) on d7 were obviously lower than those on $\mathrm{d} 1(t=7.176,14.000$ and 3.089; all $p<.001$ and .01). See Table 1, Figures 4-6 for details.

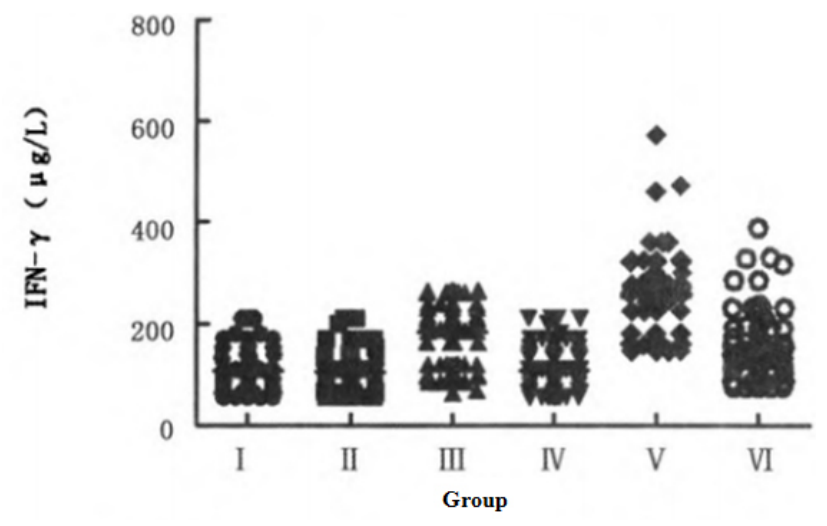

Figure 4. The expression of IFN- $\gamma$ in the serum

IFN- $\gamma$ stands for interferon $\gamma$; I stands for the normal group on $d 1$; II stands for the normal group on d7; III stands for the group (APACHE II $\leq 20$ ) on dI; IV stands for the group (APACHE II $\leq$ 20) on d7; V stands for the group (APACHE II > 20) on d1; VI stands for the group (APACHE II > 20) on $d 7$.

3.5 The analysis in the correlation of TLR4 $\mathrm{mRNA}$ with IFN- $\gamma$, IL-23 and PCT

According to the correlation analysis, it was found that, on $\mathrm{d} 1$, the correlation coefficients of TLR4 mRNA with IFN- $\gamma$ 
and IL-23 in the group (APACHE II $\leq 20$ ) and the group (APACHE II > 20) were $0.949 \& 0.957$ and $0.9149 \& 0.910$, indicating that TLR4 mRNA was significantly correlated with IFN- $\gamma$ and IL-23 (all $p<.0001$ ). On d7, the correlation coefficients of TLR 4 mRNA with IFN- $\gamma$ and IL-23 in the group (APACHE II $\leq 20$ ) and the group (APACHE II $>20$ ) were $0.1162 \& 0.110$ and $-0.1751 \& 0.040$, indicating that TLR4 mRNA was not correlated with IFN- $\gamma($ all $p>.05)$.

\subsection{The diagnostic value of TLR4 $\mathrm{mRNA}$, IFN- $\gamma$ and IL-} 23 in predicting sepsis

The data on TLR4 mRNA, IFN- $\gamma$ and IL-23 on d1 after admission were used to calculate ROC curve, and areas under curve (AUC) of TLR4 mRNA, IFN- $\gamma$, IL-23, PCT and CRP were $0.975,0.952,0.992,0.866$ and 0.894 , indicating a remarkable diagnostic sensitivity. The best cut-off value of TLR4 mRNA at baseline was $891.6 \mu \mathrm{g} / \mathrm{L}$, with a sensitivity of $100 \%$ and a diagnostic specificity of $57 \%$. The best cut-off value of IFN- $\gamma$ at baseline was $84.5 \mu \mathrm{g} / \mathrm{L}$, with a sensitivity of $100 \%$ and a diagnostic specificity of $57 \%$. The best cut-off value of IL-23 at baseline was $861.0 \mu \mathrm{g} / \mathrm{L}$, with a sensitivity of $100 \%$ and a diagnostic specificity of $97 \%$. See Figure 7 and Table 1 for details.

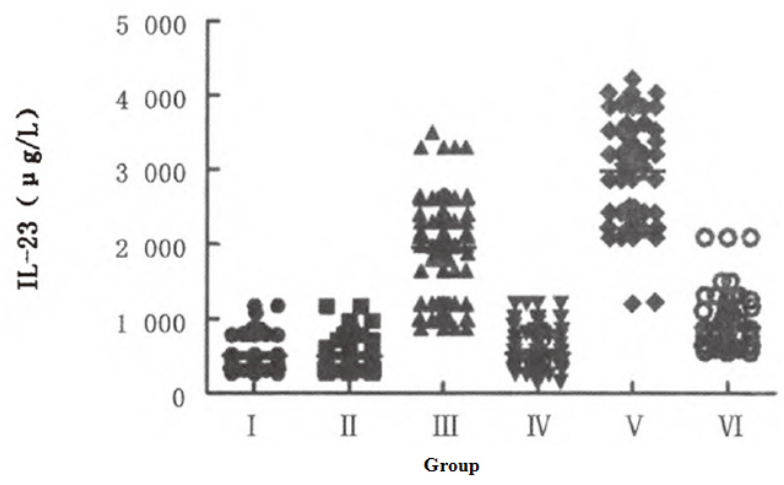

Figure 5. The expression of IL-23 in the serum I stands for the normal group on d1; II stands for the normal group on d7; III stands for the group (APACHE II $\leq 20)$ on d1; IV stands for the group (APACHE II $\leq 20)$ on $d 7 ; V$ stands for the group (APACHE II > 20) on d1; VI stands for the group (APACHE II > 20) on $d 7$.

Table 1. ROC values of different indicators in patients with sepsis

\begin{tabular}{lllll}
\hline Indexes & Cut-off Point $(\boldsymbol{\mu g} / \mathbf{L})$ & AUC & Sensitivity (\%) & Specificity $(\%)$ \\
\hline TLR4 mRNA & 891.60 & 0.975 & 100 & 57 \\
IFN- $\gamma$ & 84.50 & 0.952 & 100 & 57 \\
IL-23 & 861.00 & 0.992 & 100 & 97 \\
PCT & 0.83 & 0.866 & 85 & 100 \\
CRP & 8.25 & 0.894 & 97 & 50 \\
\hline
\end{tabular}

Note. ROC stands for receiver operating characteristic; AUC is for area under the curve.

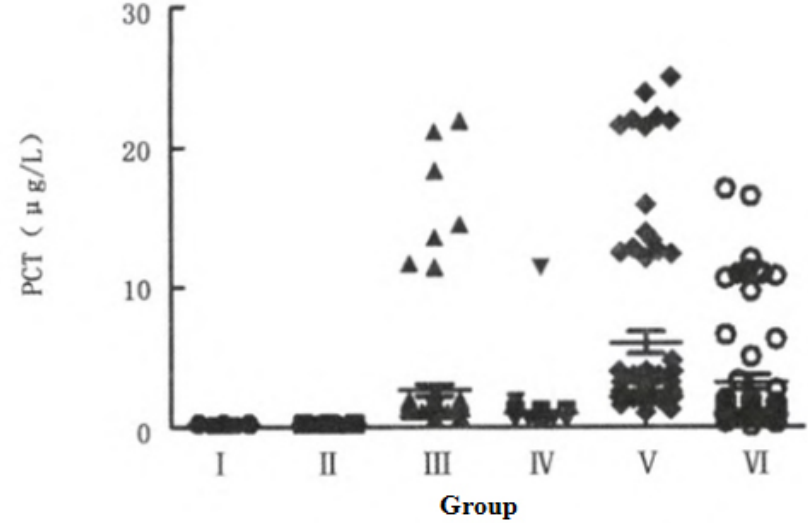

Figure 6. The expression of PCT in the serum

PCT stands for procalcitonin; I stands for the normal group on d1; II stands for the normal group on d7; III stands for the group (APACHE II $\leq 20)$ on d1; IV stands for the group (APACHE II $\leq$ 20) on d7; V stands for the group (APACHE II > 20) on d1; and VI stands for the group (APACHE II > 20) on d7.

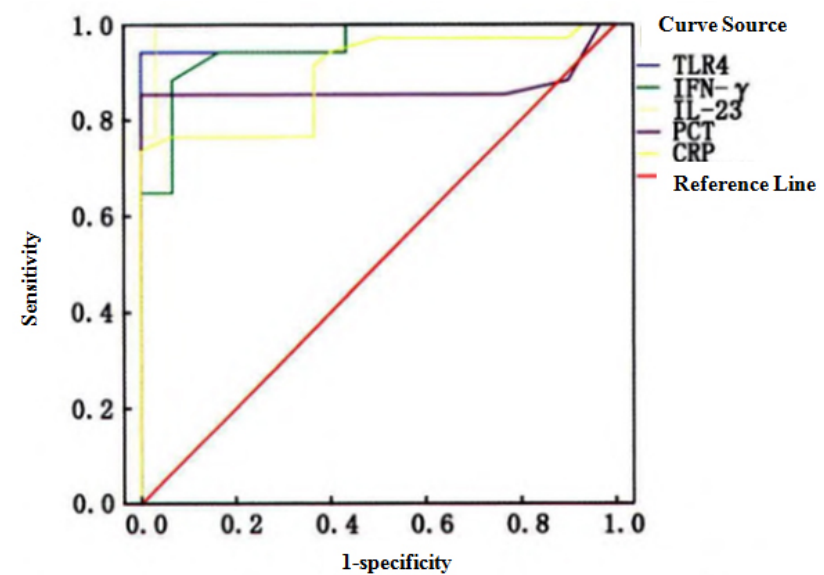

Figure 7. ROC curve of TLR4, IFN- $\gamma$, IL-23, PCT and CRP in the subjects on $\mathrm{d} 1$ after admission TLR4 stands for toll-like receptor 4; IFN- $\gamma$ stands for interferon $\gamma$; $P C T$ stands for procalcitonin; CRP stands for $C$-reactive protein; ROC stands for receiver operating characteristic. 


\section{DiscuSSION}

Gene polymorphism refers to SNPs resulted from the change of bases in gene sequence. It is commonly seen in genetic variation. SNPs can lead to the following results: the change of base sequence results in the change of its translated proteins, generating the bodily functions, such as stress susceptibility, clinical phenotype and modified drug reactions; no functional change due to no effects on protein amino acid sequences. ${ }^{[3,4]}$ Some researches have shown that abnormal inflammatory reactions caused by TLR-mediated signal transduction pathway play an important role during the seizure of sepsis, ${ }^{[5]}$ therefore, the gene polymorphism of TLRs can change the susceptibility to sepsis and the severity. ${ }^{[3,4]}$ As SNPs of TLRs lead to the change of extracellular domain and affect the productive level of inflammatory factors, the extent of TLR-mediated inflammatory reactions is changed consequently. ${ }^{[5]}$ There are a large number of researches on SNPs of TLR4 in G- bacteria-mediated sepsis, especially the researches on the replacement of TLR4 Asp299Gly aspartic acid by glycine and the replacement of Thr399Ile threonine by isoleucine. ${ }^{[6,7,9]}$ The researches from Lorenz et al. ${ }^{[6]}$ and Agnese et al. ${ }^{[7]}$ have shown that the SNPs of TLR4 can affect the occurrence, severity and prognosis of sepsis. Some reports have also indicated that the gene polymorphism of TLR4 Asp299Gly and Thr399Ile are not related with sepsis. $^{[8,10]}$ This research is aimed at TLR4 Asp299Gly loci from sepsis patients of Han nationality. The gene sequencing and the corresponding loci enzyme digestion results show no gene polymorphism of TLR4 Asp299Gly, which is similar with the research from Ahren et al. ${ }^{[8,10]}$ It may be due to the limited number of the cases in this research.

TLR-mediated inflammatory signal transduction pathway plays an important role in the immune responses. Some researches have shown that TLR4 is activated after pathogen infection, and then it mediates systemic immune responses while inducing protective immune responses. TLR4 has a significant meaning in the occurrence and development of sepsis, and the early monitoring of the expression level can help timely make a diagnosis and evaluate the severity of the disease. ${ }^{[5,9]}$ TLR4 are mainly distributed in monocytes. ${ }^{[5]}$ Therefore, mononuclear cells were used in this research due to increased expression. TLR4 mRNA expression is monitored in the patients with sepsis. APACHE II (taking 20-score as the boundary) was used to grade the severity of sepsis, with the results showing that, the expression of TLR4 mRNA is increased with the rise of APACHE II score, and its level is directly related with the severity of sepsis. The research results indicate that inflammatory responses and the severity of sepsis are related with TLR4 in the peripheral blood, and TLR4 can be used as an important indicator for the diagno- sis, evaluation and treatment of sepsis. This research will provide an important help for the further intensive study of the pathogenic mechanism of sepsis, which is identical to the previous research results. ${ }^{[6,7,9]}$

The occurrence of sepsis is related to the TLR2-TLR4 comediated transmembrane signal transduction pathway, which is activated by the process that TLR4 identifies LPS and leads to the waterfall release of unbalanced inflammatory factors. ${ }^{\left[{ }^{[9}\right.}$ IL-23, a heterodimeric cytokine which can cause inflammation, is a member of IL-12 family. It is formed by the disulphide bond of a sigmasubunit of p19 and p40. As a proinflammatory factor, IL-23 participates in the differentiation, development and proliferation of immune cells, playing an important role in the regulation of inflammatory responses. IL-23 can act on natural killer cells to induce the generation of IFN- $\gamma$; or act on memory T lymphocytes to generate a large number of proinflammatory mediators IL-17. ${ }^{[11]}$ In this research, it is found that there are statistically significant differences in the expression levels of IFN- $\gamma$ and IL-23 among these three groups on $\mathrm{d} 1$ (all $p<.01$ ); on $\mathrm{d} 7$, there is a statistically significant difference between the normal group and the group (APACHE II $>20)(p<.01)$, as to other comparisons between groups, there are not statistically significant differences (all $p>.05$ ). It is indicated that the levels of IFN- $\gamma$ and IL-23 are obviously lower in sepsis patients with the prolonging of treatment time, which can be considered as an indicator for the change trend of the disease. It is similar with the research reported by Gordon. ${ }^{[11]}$ It is also found in this research that the expression of TLR4 mRNA in sepsis patients is remarkably correlated to the expression of IFN- $\gamma$ and IL-23 on d1 (all $p<.0001$ ), however, on d7, the expression of TLR4 mRNA is not correlated to the expression of IFN- $\gamma$ and IL-23 (all $p>.05$ ). It is indicated that the expression of TLR4 mRNA is synchronous with the expression of IFN- $\gamma$ and IL-23 in the early period of sepsis. Nevertheless, the negative results remain to be confirmed by the expanded number of cases. Currently, typical experimental indicators for evaluating the severity of infection are CRP and PCT, but CRP cannot differentiate the type of inflammation. PCT is the precursor of calcitonin, and its concentration will be upregulated when bacterial infection happens. It is of diagnostic value to inflammation caused by bacterial infection. ${ }^{[12]}$ The expression of CRP and PCT is monitored in this research, indicating that the expression of TLR4 mRNA is synchronous with the increase of IFN- $\gamma$ and IL-23. It is confirmed that the above indicators have an identical clinical significance to the typical indicators, which is similar with the above researches. ${ }^{[12]}$

ROC curve can be used to evaluate the correlation of diagnostic sensitivity and specificity, and AUC is positively 
correlated to the diagnostic value. ${ }^{[13]}$ ROC curve analysis is made to the expression results of TLR4 mRNA, IFN- $\gamma$, IL-23, PCT and CRP on d1, and the corresponding AUC values are $0.975,0.952,0.992,0.866$ and 0.894 . It can be seen that the AUC values of TLR4 mRNA, IFN- $\gamma$ and IL23 are higher than those of PCT and CRP. In this research, the best cutoff value of TLR4 mRNA at baseline was 891.6 $\mu \mathrm{g} / \mathrm{L}$, with a sensitivity of $100 \%$ and a diagnostic specificity of $57 \%$. The best cutoff value of IFN- $\gamma$ at baseline was 84.5 $\mu \mathrm{g} / \mathrm{L}$, with a sensitivity of $100 \%$ and a diagnostic specificity of $57 \%$. The best cutoff value of IL-23 at baseline was 861.0 $\mu \mathrm{g} / \mathrm{L}$, with a sensitivity of $100 \%$ and a diagnostic specificity of $97 \%$. It is indicated that these three indicators have a high value in the early diagnosis of sepsis. It is concluded that TLR4 mRNA, IFN- $\gamma$ and IL-23 can be used as markers for the early diagnosis, with a higher efficiency than PCT and CRP.

\section{CONFLICTS OF INTEREST DisClOSURE}

The authors declared no conflicts of interest.

\section{REFERENCES}

[1] Javed A, Guirgis FW, Sterling SA, et a1. Clinical predictors of early death from sepsis. J Crit Care. 2017; 42: 30-34. PMid:28668774. https://doi.org/10.1016/j.jcrc.2017.06.024

[2] Bhattacharjee P, Edelson DP, Churpek MM. Identifying patients with sepsis on the hospital wards. Chest. 2017; 151(4): 898-907. PMid:27374948. https://doi.org/10.1016/j.chest. 2016.0 6.020

[3] Plantinga TS, Ioana M, Alonso S, et al. The evolutionary history of TLR4 polymorphisms in Europe. J Innate Immun. 2012; 4(2): 168175. PMid:21968286. https://doi .org/10.1159/000329492

[4] Thurow HS, Sarturi CR, Fallavena PR, et al. Very low frequencies of Toll-like receptor 2 supposed-2029T and 2258A (RS5743708) mutant alleles in southern Brazilian critically ill patients: would it be a lack of worldwide-accepted clinical applications of Toll-like receptor 2 variants? Genet Test Mol Biomarkers. 2010; 14(3): 405-419. PMid:20578945. https://doi.org/10.1089/gtmb.2009.0169

[5] Kagan JC, Medzhitov R. Phosphoinositide-mediated adaptor recruitment controls Toll-like receptor signaling. Cell. 2006; 125(5): 943955. PMid:16751103. https://doi.org/10.1016/j.cell. 200 6.03 .047

[6] Lorenz E, Mira JP, Frees KL, et al. Relevance of mutations in the TLR4 receptor in patients with gram-negative septic shock. Arch Intern Med. 2002; 162 (9): 1028-1032. PMid:11996613. https: //doi.org/10.1001/archinte.162.9.1028
[7] Agnese DM, Calvano JE, Hahm SL, et al. Human Toll-like receptor 4 mutations but not CD14 polymorphisms are associated with an increased risk of gram-negative infections. J Infect Dis. 2002; 186(10): 1522-1525. PMid:12404174. https://doi.org/10.108 $6 / 344893$

[8] Schroder NW, Schumann RR. Single nucleotide polymorphisms of Toll like receptors and susceptibility to infectious disease. Lancet Infect Dis. 2005; 5(3): 156-164. https://doi .org/10.1016/S1 473-3099 (05) 01308-3

[9] Qi M, Yang J. Research progress on the correlation of TLR4 gene polymorphism with sepsis. Journal of Clinical Pulmonary Medicine. 2014; 19(1): 146-147, 152.

[10] Ahren P, Kattner E, Kohler B, et al. Mutations of genes involved in the innate immune system as predictors of sepsis in very low birth weight infants. Pediatr Res. 2004; 55(4): 652-656. PMid: 14739370. https://doi.org/10.1203/01.PDR.0000112100.61253.85

[11] Gordon MA. Salmonella infections in immunocompromised adults. J Infect. 2008; 56(6): 413-422. PMid:18474400. https://doi .or $\mathrm{g} / 10.1016 / \mathrm{j} \cdot \mathrm{j}$ inf .2008 .03 .012

[12] Maruna P, Nedelnikovd K, Gtirlich R. Physiology and genetics of procalcitonin. Physiol Res. 2000; 49(Suppl 1): S57-S61.

[13] Wu J, Hu L, Zhang G, et al. Accuracy of presepsin in sepsis diagnosis: a systematic review and meta-analysis. PLoS One. 2015; 10(7): e0133057. PMid:26192602. https://doi.org/10.1371/journa 1. pone. 0133057 